\title{
Nanostructures of 2d Transition Metal Dichalcogenides for Hydrogen Generation under Alkaline Conditions: From Theoretical Models to Practical Electrocatalysts
}

Hydrogen has been considered as the cleanest renewable energy and the ideal alternative to fossil fuels. Electrocatalytic Hydrogen Evolution Reaction (HER) via water splitting also plays an indispensable role in high-efficiency energy con- version. Compared with the well-investigated acidic HER, the relatively slow kinetics and unclear mechanism of HER in alkaline environ- ments largely make the design of electrocatalysts a trial-and-error process, retarding the scalable development of efficient, sustainable hydrogen production. Furthermore, two-dimensional tran- sition metal dichalcogenides (2D TMDs) have been demonstrated to be promising acidic/ alkaline HER catalysts in water electrolysis due to their outstanding atom-level thickness and surface-based properties.

Keywords: alkaline HER; 2D TMDs; electrocata- lysis; surface science; structure-property correlation.

Classification: FOR Code: 291899

Language: English

London

Journals Press
LJP Copyright ID: 925652

Print ISSN: 2631-8490

Online ISSN: 2631-8504

London Journal of Research in Science: Natural and Formal 



\title{
Nanostructures of 2d Transition Metal Dichalcogenides for Hydrogen Generation under Alkaline Conditions: From Theoretical Models to Practical Electrocatalysts
}

\author{
Zhexu Xi
}

\section{ABSTRACT}

Hydrogen has been considered as the cleanest renewable energy and an ideal alternative to fossil fuels. Electrocatalytic Hydrogen Evolution Reaction (HER) via water splitting also plays an indispensable role in high-efficiency energy conversion. Compared with the well-investigated acidic HER, the relatively slow kinetics and unclear mechanism of HER in alkaline environments largely make the design of electrocatalysts a trial-and-error process, retarding the scalable development of efficient, sustainable hydrogen production. Furthermore, two-dimensional transition metal dichalcogenides (2D TMDs) have been demonstrated to be promising acidic/ alkaline HER catalysts in water electrolysis due to their outstanding atom-level thickness and surface-based properties. To minimise the gap between fundamentals and practical applications of alkali-active electrocatalysts, a class of $2 D$ ultrathin nanomaterials show an infusive potential in identifying related key descriptors and principles. In this article, a general overview based on the principles of HER is presented, especially key parameters for evaluating the activity. Next, according to the basics of HER, the controversial mechanism of the alkaline HER process is comprehensively discussed, especially the detailed comparison with the acidic HER in three aspects: proton donors, energy barriers and roles of active sites. Then, modulation strategies of $2 D$ TMDs for HER in electrocatalysis are analysed together with the theoretical calculations, electrochemical experiments and surface modification. Finally, an overall perspective of the rational design of highly efficient electrocata- lysts under alkaline solutions in water splitting system is proposed.

Keywords: alkaline HER; 2D TMDs; electrocatalysis; surface science; structure-property correlation.

Author: Bristol Centre for Functional Nanomaterials, University of Bristol, Bristol, UK.

\section{INTRODUCTION}

In the 21st century, in order to reduce the reliance on less cleaning and non-renewable energy such as fossil fuels, hydrogen has been gradually widely developed as an ideal, eco- friendly source due to its high energy density and nontoxicity ${ }^{[1][2]}$. To achieve the efficient electric-tochemical energy conversion with only water produced, water electrolysis (also known as electrochemical water splitting) is also consid- ered to be a main environmentally friendly pathway for hydrogen generation ${ }^{[3]}$. HER, one of the halfreactions in the water splitting process, occurs at anode-electrolyte interfaces. Its performance depends primarily on the catalytic reactivity of anode electrode materials as electrocatalysts ${ }^{[4][5]}$. Thus, it is of great impor- tance to explore further two questions: How do electrolyte solutions affect the HER activity and kinetics? What is the relationship between the electrocatalyst nanostructures and electroca- talytic performance?

Specifically, one of the inevitable challenges in electrocatalysis is to identify the relationship between microscopic dynamics of intermediate species or absorbed states and macroscopic 
kinetics. Also, the apparent rate constant in electrocatalytic reactions usually counts on the extrinsic electrocatalyst-hydrogen interactions at electrocatalyst-electrolyte interfaces and the intrinsic electronic properties of the specific electrocatalyst. Accordingly, the electrolyte and the electrocatalyst always act together to determine the behavior of adsorbed intermediates, energy barriers and overpotential/activation energies via these extrinsic and intrinsic features $^{[4][6]}$. HER is just an ideal reaction model to value the inner interdepe- ndence from a nanoscale world to real-world applications, so HER plays an momentous role in narrowing the gap between theoretical framework on surface electrolysis and practical design of electrocatalysts $^{[7]}$. For instance, the combination of electrochemical measurements and surface characterisation successfully brings a wide-range relationship between surface physicochemical properties and catalytic activ- ity ${ }^{[8]}$; Similarly, in terms of the theoretical calculations, a direct quantitative model can be built between the electronic structure and appa- rent activity of an electrocatalyst ${ }^{[9]}$.

The above comprehensively investigated research has largely boosted the in-depth understanding of HER in electrocatalysis, especially in acidic medium $\left(2 \mathrm{H}^{+}+2 \mathrm{e}^{-} \rightarrow \mathrm{H}_{2}\right)$, including how to identify the active sites, and how to evaluate the catalytic activity on various electrode materials for better design. Meanwhile, the alkaline HER mechanism is still under debate, mainly behind the discovery that the kinetics (regarding the higher exchange current densities $j_{o}$ ) in acid is 2-3 orders of magnitude faster than in alkali, thus suggesting a higher overpotential to push forward the reaction ${ }^{[5,10,11]}$. The debate mainly includes:

1) the nature of $\mathrm{pH}$ dependence on the HER performance ${ }^{[11]}$;

2) three core descriptors: water dissociation ${ }^{[12]}$, Hydrogen Binding Energy $(\mathrm{HBE})^{[13]}$, and surface $\mathrm{H}^{*} / \mathrm{OH}^{*}$ exchange process ${ }^{[14]}$.

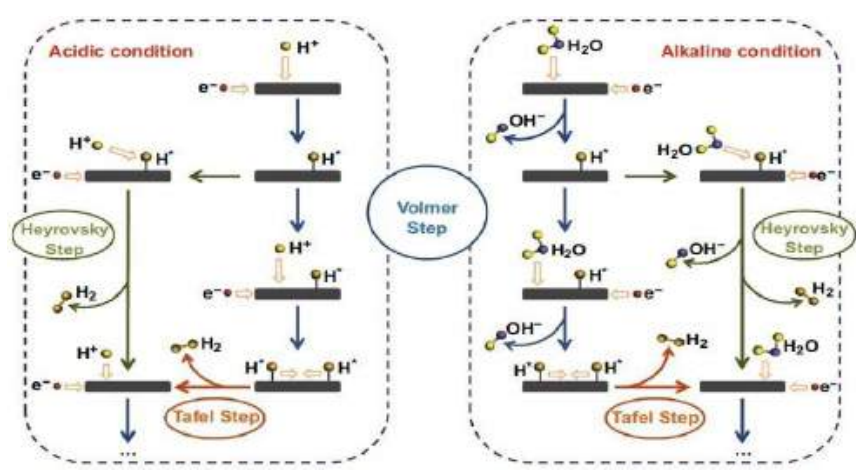

Figure 1: Schematic mechanisms of HER under acidic (left) and alkaline (right) conditions (with additional participation of dissociated water molecules as $\mathrm{H}^{*}$ donors), both containing two crucial steps: adsorption of hydrogen atoms $\left(\mathrm{H}^{*}\right)$ on the active centers of electrode surface (the Volmer Step), and then generation of $\mathrm{H}_{2}$ molecules by combination with another adsorbed $\mathrm{H}^{*}$ (the Tafel Step) or with a proton from solution (the Heyrovsky Step) $)^{[15]}$

From Fig. 1, although the HER electrocatalysts act in the similar pathways in acidic and alkaline medium, the sluggish rate in alkali stems from the additional water dissociation $\operatorname{step}^{[15]}$. Consequently, to design novel, tailor-made materials for HER enhancement, these debates become emerging desires to explore how the principles of HER can be illustrated under the condition of a universal $\mathrm{pH}$ range (design from the science perspective), how more electrocatalytically active sites can be exposed via modified surfaces or distribution of atoms (design from the combined perspective between science and engineering), and then how morphological features can be better utilised for better apparent HER activity (design from the engineering perspective).

Recently, nanostructured metallic materials with low dimensionality has captured extensive atte- 
ntion in the rational design, fabrication and characterisation of ideal HER electrocatalysts due to their high atom utilisation efficiency with strengthened electrochemical hydrogen production activity ${ }^{[16]}$. Particularly, compared with other kinds of widely applied HER electrocatalysts, 2D layered TMDs nanosheets (NSs)/nanoplates has exhibited fabulous electrocatalytic performance because of their fast electron mobility, high specific surface area, excellent surface unsaturated atoms as active sites and subsequent diversified synthesis methods ${ }^{[17][18]}$. Thus, 2D TMDs have great potential in becoming a cost-effective and highly efficient electrocatalysts for better understanding of the alkaline HER mechanisms.

Generally, it's of great value to combine theoretical calculations and experimental studies together for the rational design of 2D TMDsbased HER electrocatalysts under alkaline solutions. Here, the fundamental principles of HER process are firstly presented as well as emphasis on recent understanding and evaluation of alkaline HER. Next, the outline of 2D TMDs is highlighted to signify the structure-, surface- and morphology-related roles for connections with key activity descriptors of alkaline hydrogen generation. Then, more importantly, this systematic knowledge is extended to a broader level, which entails the design rules of practically well-defined electrocatalysts by discussing the correlations between the nanostructures of TMDs and the contributions of active sites. Finally, a personal perspective on the future feasibility in TMDs-based alkali- active electrocatalysts is proposed.

\section{FUNDAMENTALS OF HER UNDER ALKALINE CONDITIONS}

\subsection{The role of $\mathrm{pH}$ in HER}

Compared with the acidic and alkaline HER processes (Fig. 1), the sluggish rate in alkaline Medium (about 2-3 orders of magnitude lower activity than in acid medium) may originate from the additional dissociation of water to provide adsorbed $\mathrm{H}^{*}$ in the Volmer reaction, thereby leading to the low concentration of $\mathrm{H}^{*}$. As water molecules become the sole proton donors in alkaline environments, the slow water dissociation step inevitably causes the slow rates in subsequent steps.

This explanation has been implicitly supported by a system of HER tests with different metallic electrodes and varying $\mathrm{pH}$ conditions, from acidic $(\mathrm{pH}=1-4)$, to neutral $(\mathrm{pH}=4-11)$, and to alkaline ( $\mathrm{pH}=11-14)$ (Fig.2) ${ }^{[19]}$. Besides the demonstration of a strikingly weakened HER activity in alkali, the increasing $\mathrm{pH}$ up to $\mathrm{pH}=4$ presents a pure diffusion limiting current different from the typical polarisation profile, accordingly revealing the characteristic change of metallic surfacespecific electrode potential. This discovery signifies the key influential factors of HER process is the mass transport of $\mathrm{H}_{3} \mathrm{O}^{+}$(hydrogen intermediate species), not the charge transfer. Also, when the electrolyte solutions become neutral or alkaline, the polarisation processes behind the current-potential curves of different electrocatalysts are clearly free from the change of $\mathrm{pH}$ values, indicating that currents is intimately related to $\mathrm{pH}$-independent transformation from water to hydrogen molecules ${ }^{[19][20]}$. 

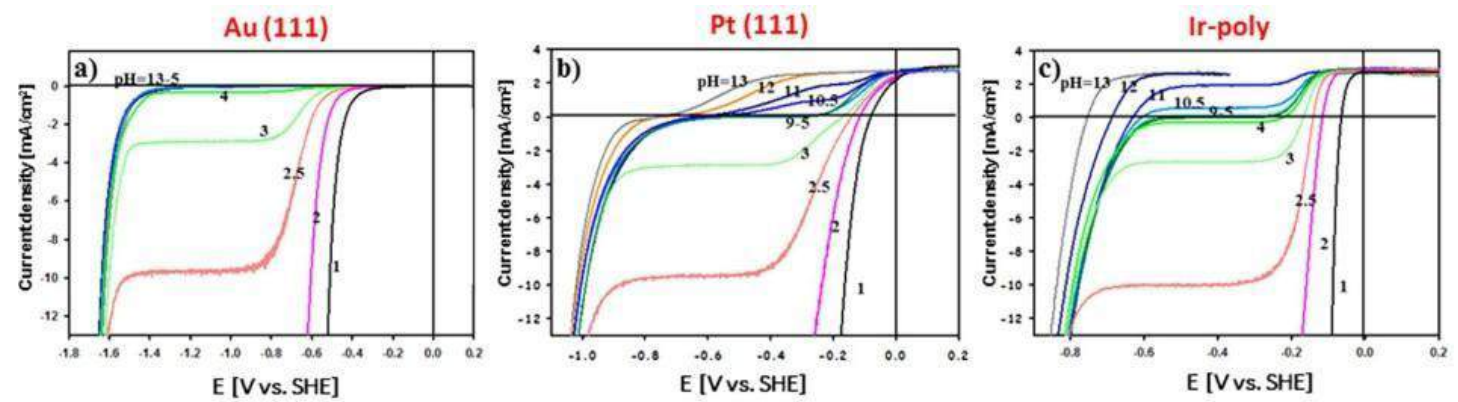

Figure 2: Polarisation (current-potential) curves for three metallic HER electrocatalysts: a) Au(111), b) $\mathrm{Pt}(111)$, and c) Ir-poly. Experimental conditions: $0.1 \mathrm{M} \mathrm{NaClO}_{4}$ as solvents, purged with $\mathrm{H}_{2}$, addition of $\mathrm{NaOH}$ or $\mathrm{HClO}_{4}$ for $\mathrm{pH}$ adjustment. Testing conditions: rotation rates of $1600 \mathrm{rpm}$ and sweep rate of $50 \mathrm{mV} / \mathrm{s}^{[19]}$.

Overall, HER activity is seemingly pH-dependent, but the slow kinetics of alkaline HER has been demonstrated not to be entirely interpreted by the $\mathrm{pH}$ values near the catalyst surface. More accurate and comprehensive explanations need to be highlighted for bridging the gap between the underlying alkaline HER mechanisms and the practical design principles of electrocatalysts. Three reasonable theories are mentioned based on the current investigations: water dissociation theory, hydrogen binding energy (HBE) theory and $\mathrm{H}^{*} / \mathrm{OH}^{*}$ or interface water transfer theory.

\subsection{Water dissociation: the rate-determining step?}

As mentioned in 2.1., the kinetics of alkaline hydrogen production is strictly restricted by the additional water dissociation step. Different from the high concentration of $\mathrm{H}^{*}$ with $\mathrm{H}^{*}$-rich surfaces in acidic environments, water is the sole proton donor for the Volmer Reaction of alkaline/neutral HER, thus producing an extra tremendous energy barrier for cleaving $\mathrm{H}-\mathrm{O}-\mathrm{H}$ bond and generating hydrogen intermediates. As a result, water dissociation can be regarded as the rate-determining step of alkaline HER. The overall microscopic process in basic media was perfectly verified by Subbaraman et al. that $\mathrm{Ni}(\mathrm{OH})_{2}$ clusters prompted the HER kinetics and activity by improving water dissociation for $\mathrm{H}^{*}$ generation near the Pt surface ${ }^{[21]}$.

Nonetheless, the water dissociation theory still cannot receive the universal acceptance in the electrocatalytic community because the experime- nts are conducted on the surface with high surface area, not in agreement with the ideal computational model surfaces.

\subsection{Hydrogen Binding Energy: applicable under a universal range of $\mathrm{pH}$ ?}

Considering the similar HER reaction pathways in acid and alkali, the HER catalytic performance is regarded to be closely linked with $\mathrm{HBE}$ in all $\mathrm{pH}$ conditions, which can be measured on the electrode interface ${ }^{[13][22]}$. HBE is reckoned to be correlated with HER kinetics, as change of hydrogen underpotential adsorption/desorption $\left(\mathrm{H}_{\text {upd }}\right)$ peak positions is related to $\mathrm{H}_{2}$ production rates. Another evidence suggests that too intense HBE results in a high energy barrier for water activation, naturally triggering sluggish kinetics of

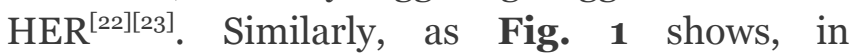
nanostru- ctured electrocatalysts, weaker hydrogen adsor- ption capacities bring greater exchange current densities ${ }^{[19][23]}$.

However, HBE is an intrinsic parameter of catalyst, so it is pH-independent; accordingly, an assumption was proposed that HER kinetics depends on the net effect of HBE and water adsorption, and then was further demonstrated by the Goddard group that water at interfaces have different orientation with the corresponding adsorption behavior under different $\mathrm{pH}^{[10][24]}$. Specifically, more basically soluble environments bring weaker surface adsorption of water molecules and conversely promotes the strength of $\mathrm{H}-\mathrm{O}-\mathrm{H}$ bond. 
Despite identification of the role of water adsorption at interfaces in $\mathrm{pH}-\mathrm{HBE}$ correlation, the HBE theory cannot be the sole descriptor of HER process under all $\mathrm{pH}$. The interface nanostructures of catalyst can also contribute to the HER activity. For instance, the impact of electric double layers on the HER was thoroughly studied by the Koper group that $\mathrm{H}_{\text {upd }}$ peak and the potential of zero charge (pzc) shift in the same way as the $\mathrm{pH}$ values change[25]. This indicates that the reorganisation energy can be tuned for accelerated $\mathrm{H}+\mathrm{OH}-$ migration from the surface to the bulk, thereby regulating the activation barrier for $\mathrm{H}^{*}$ adsorption and HER kinetics. Specifically, alkaline medium suggests a large reorganisation energy, thereby causing a relatively slow gas production rate.

\section{4 $\mathrm{H}^{*} / \mathrm{OH}^{*}$ transfer at interfaces: the competitive role of $\mathrm{OH}^{*}$}

The Koper's research indicates the indispens- able role of interfacial water activation and $\mathrm{H}^{*}$ adsorption for boosted HER activity. In fact, this discovery generates another viewpoint that the $\mathrm{H}^{*} / \mathrm{OH}^{*}$ adsorption in alkaline medium are always in a race with a shared surface active site, thus decreasing the hydrogen generation rate. In addition, Liu et al. verified that cleaving $\mathrm{OH}^{*}$ on catalyst surface weakens the alkaline HER kinetics on account of the absence of $\mathrm{OH}^{*}$-based interface adducts ${ }^{[26]}$. According to the two studies, although adsorption of $\mathrm{OH}^{*}$ cannot participate in the Volmer Step or affect the HER activity solely, $\mathrm{OH}^{*}$ mobility through the electric double layer does also play a part for HER enhancement by altering the $\mathrm{pH}$-dependent density of $\mathrm{OH}^{-}$and metal anions ${ }^{[19][26]}$.

Therefore, although $\mathrm{OH}^{*}$ cannot exert a direct influence on the stimulation of HER kinetics, it is still considered to have connectivity with the role of $\mathrm{OH}^{-}$and the related sluggish alkaline HER kinetic rates. More significantly, the $\mathrm{OH}^{*}$ and cations at interfaces provide a new insight into the rational design of "dual active sites" electrocatalysts. This strategy promotes the efficiency in identification of $\mathrm{H}^{*} / \mathrm{OH}^{*}$ by creating another active site for spatial separation of the two intermediates.

\subsection{Overall impact on alkaline HER}

Although massive studies have been implemented to strengthen the understanding of the internal principles of alkaline HER, especially the debate on whether extra water dissociation or $\mathrm{H}^{*}$ adsorption process acts decisively, there is still no answer regarding these issues on the atomic or molecular level. However, they all mentioned the additional activation barrier stemming from breaking the strong $\mathrm{H}-\mathrm{O}-\mathrm{H}$ bonds and extracting abundant intermediates from water molecules. Naturally, both theoretical and experimental studies suggest the net impact between the Gibbs Free Energy change of water dissociation and of $\mathrm{H}^{*}\left(\right.$ or $\mathrm{OH}^{*}$ ) adsorption as the key factors in alkaline $\mathrm{HER}^{[27]}$. These factors all determine the overall rate as well as the enthalpy entailing a series of energy barriers.

Hence, while in alkaline medium, the optimum reaction pathway is to minimise the energy barriers between the kinetic water dissociation and the thermodynamic $\mathrm{H}^{*} / \mathrm{OH}^{*}$ adsorption. However, from the aforementioned debate, the effect of electrode potential and electrolyte $\mathrm{pH}$ can be the main factors to affect the two processes. In other words, it's extraordinarily vital to construct an efficient hydrogen evolution system considering the above factors, underlying methodologies behind the controversial theories as well as experimental data.

\section{ADVANTAGES OF 2D TMDS FOR CLEARER UNDERSTANDING OF

$$
\text { ALKALINE HER }
$$

\subsection{Advantages of nanostructured 2D TMDs}

2D TMDs (usually expressed in the formula of $\mathrm{MX}_{2}$, where $\mathrm{M}=\mathrm{Mo}, \mathrm{W}, \mathrm{Ta}$ or $\mathrm{Nb}$ and $\mathrm{X}=\mathrm{S}$, Se or $\mathrm{Te}$ ), as an intriguing part in the large family of $2 \mathrm{D}$ nanomaterials, has drawn an increasing attention due to unique atomic and electronic structures with outstanding, surface-related preparation technologies and characterisation techniques[28].

Because of electrons strictly confined in the ultra-thin 2D space, especially for monolayered materials, the excellent structural and electronic properties of 2D TMDs could be generated. 
Secondly, their great in-plane covalent bond strength and atom-level thickness contribute to appealing flexibility and mechanical strength ${ }^{[29]}$. Thirdly, their high specific area can provide plentiful active sites for boosted electrocatalysis; also, a system of mature modification strategies (e.g. stress-strain effect, heteroatom doping, defect engineering, phase transformation) help expose active facets selectively for high-selectivity catalysis. Fourthly, the surface functionalisation and the aforementioned strategies could be more easily conducted to modulate the electronic states or band structures of TMDs owing to their broad surfaces with high exposure of atoms ${ }^{[29][30]}$.

\subsection{How 2D TMDs contribute to structure-activity analysis}

Because of high specific area and well tunable engineered structures as well as a system of various TMDs-targeted preparation technologies and characterising methods, 2D TMDs can be endowed with versatile electronic, physicochemical and surface properties, and also comprehensively understood considering several key features: sizes, shapes, compositions, thicknesses, surface states, defects, crystal phases, vacancies, strains and electronic states ${ }^{[6,21,29]}$. Similarly to the ideas of structure-activity correlations in acidic HER(Fig. 3), regulating these features can evidently optimise the activation barriers originating from the water dissociation and $\mathrm{H}^{*}$ adsorption and facilitates the water dissociation step, thereby improving the HER activity in alkaline environments $\mathrm{s}^{[2,4,31,32]}$.

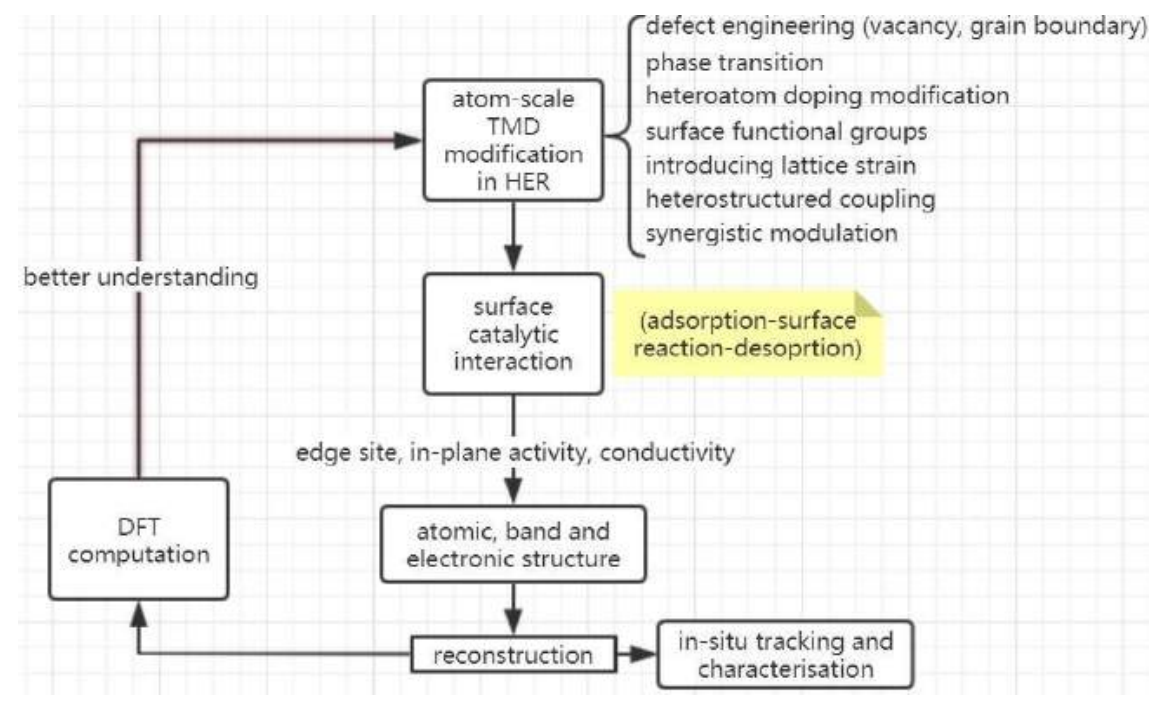

Figure 3: Schematic Principles of the Optimal Design and Modulation of TMDs-based HER Electrocatalysts in Acidic Medium ${ }^{[32]}$

To understand the structure-activity correlation more clearly, it's necessary to confirm the relationship between the surface morphology of a specific electrocatalyst and $\mathrm{H}_{2}$ production rates. Firstly, TMDs provide a huge surface to establish the links between the kinetics and any possible parameters. Besides the aforementioned $\mathrm{H}^{*} / \mathrm{OH}^{*}$ combination competition, water at catalyst-based interfaces, local internal electric field and solvent effect, surface $\mathrm{H}^{*}$ coverage in different intensities is another factor to describe the controllable kinetics of alkaline HER by impairing the HBE since the hybridization between the s state of ${ }^{*} \mathrm{H}$ and the $\mathrm{d}$ band of transition metal would shift the d-band center ${ }^{[33]}$. Secondly, in various theoretical and experimental research, the role of surface defect densities and spatial distribution are highlighted as potential active sites for HER enhancement by generating a more active and well-ordered surface $\mathrm{e}^{[5,21,28]}$. 
Consequently, the structure and morphology of TMDs provide more insights into the design of practical HER electrocatalysts. More discussions on considerations of bridging the gap between theoretical models and real-world products are required hereafter.

\section{THE RATIONAL DESIGN OF PRACTICAL TMDS-BASED ELECTROCATALYSTS IN ALKALINE HER}

\subsection{General guidelines of constructing alkali-active catalysts}

an

Although it's still unclear whether the kinetic water dissociation or the thermodynamic adsorption/desorption of activated intermediate species acts more significantly in alkaline HER, plentiful studies have established links among structural properties of TMDs, main descriptors stemming from the three controversial theories and practical efficiency in HER by analysing the involved intermediates $\left(\mathrm{H}_{3} \mathrm{O}^{+}, \mathrm{OH}^{-}\right.$, and other cations/anions at interfaces) with accompanying altered activation barriers ${ }^{[6,7,9,21-23,38]}$. From materials science perspectives, because $\mathrm{H}^{+}$and $\mathrm{OH}^{-}$are both participators in the alkaline HER process, it's vital to optimally balance the abilities of water dissociation and $\mathrm{H}^{*}$ adsorption. Accordingly, two feasible suggestions are mentioned: reducing the energy barrier thermodynamically and modulating the catalyst-hydrogen interaction kinetically. In addition, note that great physical properties of TMDs (e.g. large surface area, mechanical strength and high electron mobility) largely influence the durability and activity of catalysts ${ }^{[8,13,34]}$.

Besides the thermodynamic and kinetic factors, the differentiation of theoretical and real surfaces of nanomaterials could not be ignored. Here, the most important factor is active sites. Although the broad surface endows TMDs with large numbers of active sites, coverage of adsorbed $\mathrm{H}^{*} / \mathrm{OH}^{*}$ could decrease the densities of available sites on the surface ${ }^{[19][35]}$.

Moreover, not all defect states might be active centers for HER optimisation, so the tailored surface morphology with well-defined arrangement of defects could have a different impact on HER compared with the ideal surface $^{[23][36]}$.

\subsection{The proton donor and generation of energ barrier from activation}

All of the three theories have emphasised a tailor-made reaction pathway to connect the change of activation barriers with two thermodynamic states entailing water molecules, $\mathrm{H}^{*}$, $\mathrm{OH}^{*}$ and other potential cations/anions at catalyst-electrolyte interfaces. This is closely related to the dissociated water for donating protons and the adsorbed hydrogen intermediates for formation of the activated hydrogen-catalyst complexes $^{[21][33]}$.

No matter the origin of $\mathrm{H}^{*}$ is $\mathrm{H}_{2} \mathrm{O}$ or $\mathrm{H}_{3} \mathrm{O}^{+}$, the $\mathrm{H}^{*}$ discharge process in alkaline media is too slow to be tracked experimentally by the water dissociation mechanism ${ }^{[37]}$. The drastic change presented in the polarisation curve is the total effect of $\mathrm{H}^{*}, \mathrm{OH}^{*}$, and other existing proton donors in electrolytes like $\mathrm{HSO}_{4}^{-}$and $\mathrm{HCO}_{3}{ }^{-[38]}$. Thus, the additional water dissociation step could tune the subsequent intermediate formation and even be the rate-determining step to facilitate the entire kinetics.

As for thermodynamic adsorption/desorption processes, to promote the subsequent reactions, the bond of $\mathrm{H}^{*}$ and $\mathrm{OH}^{*}$ bound to the catalyst surface is required not to be too strong or too weak. The TMD-based electrocatalysts should possess bi-functionality to keep a balanced binding strength at interfaces as well as the proper degree of surface coverage between two kinds of intermediate species ${ }^{[19]}$. Accordingly, proper partition of surface bonding with $\mathrm{H}^{*}$ and $\mathrm{OH}^{*}$ spectators could tune the activation barrier, thereby optimising the adsorption/ desorption process and achieving the enhanced HER efficiency. 
4.3 Well-defined TMDs nanosurface with well-organised active sites: connections between ideal models and practical catalysts

\subsubsection{Different surface of ideal and realistic TMDs-based electrocatalysts}

The aforementioned various kinds of species and the hydrogen/hydroxyl-catalyst interactions are highly sensitive to the local atom-thin structure of TMDs, triggering the structurally sensitive HER process $^{[23][36]}$. In addition, nanostructures with high surface area are urgently required to enhance the specific activity for the practical use of energy conversion devices. Note that the realistic existence of edges and defects can be potential catalytically active centers to modulate the electronic structures or band levels of TMDsbased electrocatalysts. More importantly, the spatial orientation and distribution of surface defects can tune the alkaline HER activity by regulating the interface interaction of $\mathrm{H}^{*} / \mathrm{OH}^{*}$ spectators $^{[30][37]}$.

Therefore, both the theoretical insights of surface-sensitive dynamics should be extended to a more real-life level in agreement with the experimental results of TMDs-related electrocatalysis. This link could be ascribed to a welldefined nanoscale and ordered nanostructure with great crystallinity derived from the systematic calculation considering morphological features on the catalyst surface like facets, edges, step sites and corners.

\subsubsection{Core idea: optimising the availability of available active sites}

For the purpose of strengthening the electrocatalytic HER performance as well as achieving the expected consistency between the macroscopic HER activity and the microscopic 2D structural factors, diversified nanosurfaces are constructed via versatile modification methods such as heteroatom doping, defect/disorder engineering and vertically arrayed heterostructure construction ${ }^{[38]}$. With these methods, the surface adsorption energetics is changed by altering the electronic structures and simultaneously, more available active sites could be exposed or created, forming a type of synergistic coordination. Further, a more in-depth insight has been proposed and afterwards demonstrated that the entire alkaline HER activity is determined by a combined effect of $\mathrm{H}^{*}$ adsorption and $\mathrm{OH}^{*}$ desorption ${ }^{[39]}$. This opinion indicates a balance between the transition state energy of water molecules and the final state energy of $\mathrm{H}^{*} / \mathrm{OH}^{*}$ "spectator" species, and highlights the bi-functionality of active sites, also suggesting the validity of these modification methods in $2 \mathrm{D}$ TMDs-related structure-activity analysis.

Moreover, by monitoring the surface-controlled or structural parameters, the coordination environment (containing orientation, local density and distribution) and function (dependent on the categories of reaction intermediates) of active sites on the TMDs surfaces/interfaces can be precisely tuned. Theoretically, entirely different from $\mathrm{H}^{*}$ as the sole intermediate in acid, there might be a surfeit of various species in alkaline medium: $\mathrm{H}^{*}, \mathrm{OH}^{*}, \mathrm{H}_{2} \mathrm{O}$, other electrolyte-related anions/cations ${ }^{[6,8,10,12,19-21,37]}$. In other words, diversified spectators can form the bond with different kinds and strengths at interfaces, so more complicated species and energy change will be taken into account in the design and engineering of active sites.

\subsubsection{The roles of available active sites in design and engineering}

Under the principles of the experimental and theoretical considerations mentioned above, the availability of active roles plays a crucial role in the rational design of TMDs-based electrocatalysts, especially in terms of the homogeneity of electrocatalysts with tailored surfaces or nanostructures ${ }^{[19,37]}$.

Firstly, the systematic design must be built on the studies of extended interfaces. Usually, based on the introduction of active sites, the correlation between coordination environment, binding energies and practical activity can be observed to better predict an ideal geometric environment of the sites ${ }^{[40]}$. Specifically, the measured coordination number of the surface sites can be closer to the optimum value by mobilising the second nearest atoms for modified coordination of nearest atoms. 
Other similar strategies based on the formation of ordered surfaces with reduced bulk surfaces can contribute to the maximum exposure of well-organised sites as well as optimised geometric environments and altered electronic states. Further, the construction of various active sites may result in more disordered surfaces such as typically more active edge sites and relatively inert in-plane sites of TMDs, representing an extraordinarily high initial activity and stability ${ }^{[41,42]}$. The disordered surfaces can provide an efficient route for the design of alkali-active and durable electrocatalysts.

Thus, the rational design of TMDs-based electrocatalysts requires mild binding energy and a proper coordination environment, especially entailing the particle interactions at the electrolyte-catalyst interfaces, for better activity, durability and selectivity.

\section{SUMMARY AND PERSPECTIVES}

The origin of $\mathrm{pH}$-dependent hydrogen evolution kinetics is the synergistic result of $\mathrm{H}^{*}$ surface coverage, water molecules at interfaces and $\mathrm{H}^{*} / \mathrm{OH}^{*}$-catalyst bonds. The sluggish rate of HER in alkaline medium is due to the additional water dissociation step and the subsequent adsorption energetics. By providing a detailed explanation of three controversial theories and analysing the structure-activity correlations in terms of the critical descriptors stemming from the theories (source of donated protons, formation of activation barriers and active sites from the interactions at interfaces), the combinations of theoretical and experimental studies are summarised to bridge the gap between the calculated models and the real-life applications of electrocatalysts. Following the structure-descriptor-activity guidelines, TMDs with 2D well-defined nanostructures, atom-level thickness and a high specific surface area have been demonstrated to be alkali-active and stable electrocatalysts, in agreement with the theoretical induction and experimental results.

However, because of the complicated models entailing various intermediates and unpredictable energy change between thermodynamic states, a more accurate in-situ tracking and characterisation system can be developed to confirm the real-time interactions between electrocatalysts and intermediates. Note that the local environment at interfaces and the mass transport kinetics need to be highlighted as two extra important factors. Also, to make the theoretical calculations closer to experimental results, more precisely tunable crystal surfaces with nanostructures should be taken into account in terms of size, shape, defects, phase structure and crystallinity.

Finally, the role of electric double layers is mentioned, but till now, no targeted research in revealing its function in optimising the mobility of ions across the layers has been conducted. The relationship between surface properties of electrocatalysts and the layers needs a more comprehensive understanding.

\section{REFERENCES}

1. Wu, M.; Zhang, J.; He, B. -B.; Wang, H. -W.; Wang, R.; Gong, Y.-S. In-Situ Construction of Coral-Like Porous P-Doped $\mathrm{g}-\mathrm{C}_{3} \mathrm{~N}_{4}$ Tubes with Hybrid 1D/2D Architecture and High Efficient Photocatalytic Hydrogen Evolution. Appl. Catal. B: Environ. 2019, 241, 159-166.

2. Zheng, Y.; Jiao, Y.; Jaroniec, M.; Qiao, S. Advancing the Electrochemistry of the Hydrogen-Evolution Reaction through Combining Experiment and Theory. Angew. Chem. Int. Ed. 2015, 54, 52-65.

3. Zhang, J.; Wang, T.; Liu, P.; Liu, S.; Dong, R.; Zhuang, X.; Chen, M.; Feng X. Engineering Water Dissociation Sites in $\mathrm{MoS}_{2}$ Nanosheets for Accelerated Electrocatalytic Hydrogen Production. Energy Environ. Sci. 2016, 9, 2789-2793.

4. Zhou, M.; Weng, Q.; Zhang, X.; Wang, X.; Xue, Y.; Zeng, X.; Bando, Y.; Golberg, D. In Situ Electrochemical Formation of Core-Shell Nickel-Iron Disulfide and Oxyhydroxide Heterostructured Catalysts for a Stable Oxygen Evolution Reaction and the Associated Mechanisms. J. Mater. Chem. A 2017, 5, 4335-4342.

5. Miao, M.; Pan, J.; He, T.; Yan, Y.; Xia, B.; Wang, X. Molybdenum Carbide-Based Elec- 
trocatalysts for Hydrogen Evolution Reaction. Chem. Eur. J. 2017, 23, 10947-10961.

6. Strmcnik, D.; Lopes, P.; Genorio, B.; Stamenkovic, V.; Markovic, N. Design Principles for Hydrogen Evolution Reaction Catalyst Materials. Nano Energy 2016, 29, 29-36.

7. Jiao, Y.; Zheng, Y.; Jaroniecb, M.; Qiao, S. Design of Electrocatalysts for Oxygen and Hydrogen-Involving Energy Conversion Reactions. Chem. Soc. Rev. 2015, 44, 2060-2086.

8. Stamenkovic, V.; Strmcnik, D.; Lopes, P.; Markovic, N. Energy and Fuels from Electrochemical Interfaces. Nature Mater. 2017, 16, 57-69.

9. Skúlason, E.; Jónsson, H. Atomic Scale Simulations of Heterogeneous Electrocatalysis: Recent Advances. Adv. Phys. 2017, 2, 481-495. 10. Schmidt, T.; Ross Jr., P.; Markovic, N. Temperature Dependent Surface Electrochemistry on Pt Single Crystals in Alkaline Electrolytes: Part 2. The Hydrogen Evolution / Oxidation Reaction. J. Electroanal. Chem. 2002, 524-525, 252-260.

11. Jia, Q.; Liu, E.; Jiao, L.; Li, J.; Mukerjee, S. Current Understandings of the Sluggish Kinetics of the Hydrogen Evolution and Oxidation Reactions in Base. Curr. Opin. Electrochem. 2018, 12, 209-217.

12. Subbaraman, R.; Tripkovic, D.; Strmcnik, D.; Chang, K. -C.; Uchimura, M.; Paulikas, A.; Stamenkovic, V.; Markovic, N. Enhancing Hydrogen Evolution Activity in Water Splitting by Tailoring $\mathrm{Li}^{+}-\mathrm{Ni}(\mathrm{OH})_{2}-\mathrm{Pt}$ Interfaces. Science 2011, 334, 1256-1260.

13. Durst, J.; Siebel, A.; Simon, C.; Hasché, F.; Herranz, J.; Gasteiger, H. A. New Insights into the Electrochemical Hydrogen Oxidation and Evolution Reaction Mechanism. Energy Environ. Sci. 2014, 7, 2255-2260.

14. Janik, M.; McCrum, I.; Koper, M. On the Presence of Surface Bound Hydroxyl Species on Polycrystalline $\mathrm{Pt}$ Electrodes in the "Hydrogen Potential Region" (o-0.4 V-RHE). J. Catal. 2018, 367, 332-337.

15. Wei, J.; Zhou, M.; Long, A.; Xue, Y.; Liao, H.; Wei. C.; $\mathrm{Xu}, \mathrm{Z}$. Heterostructured Electrocatalysts for Hydrogen Evolution Reaction Under Alkaline Conditions. Nano-Micro Lett. 2018, 10, 75 .
16. Zhao, X.; Dai, L.; Qin, Q.; Pei, F.; Hu, C.; Zheng, N. Self-Supported 3D PdCu Alloy Nanosheets as a Bifunctional Catalyst for Electrochemical Reforming of Ethanol. Small 2017, 13, 1602970.

17. Ding, Q.; Song, B.; Xu, P.; Jin, S. Efficient Electrocatalytic and Photoelectrochemical Hydrogen Generation Using $\mathrm{MoS}_{2}$ and Related Compounds. Chem 2016, 1, 699-726.

18. Garlyyev, B.; Fichtner, J.; Piqué, O.; Schineider, O.; Bandarenka, A. S.; Calle-Vallejo, F. Revealing the Nature of Active Sites in Electrocatalysis. Chem. Sci. 2019, 10, 80608075 .

19. Strmcnik, D.; Uchimura, M.; Wang, C.; Subbaraman, R.; Danilovic, N.; van der Vliet, D.; Paulikas, A.; Stamenkovic, V.; Markovic, N. Improving the Hydrogen Oxidation Reaction Rate by Promotion of Hydroxyl Adsorption. Nat. Chem. 2013, 5, 300-306.

20. Zeng, Z.; Chang, K.; Kubal, J.; Markovic, N.; Greeley, J. Stabilization of Ultrathin (Hydroxy) Oxide Films on Transition Metal Substrates for Electrochemical Energy Conversion. Nat. Energy 2017, 2, 17070.

21. Subbaraman, R.; Tripkovic, D.; Strmcnik, D.; Chang, K. -C.; Uchimura, M.; Paulikas, A.; Stamenkovic, V.; Markovic, N. Enhancing Hydrogen Evolution Activity in Water Splitting by Tailoring $\mathrm{Li}+-\mathrm{Ni}(\mathrm{OH}) 2-\mathrm{Pt}$ Interfaces. Science 2011, 334, 1256-1260.

22. Sheng, W.; Zhuang, Z.; Gao, M.; Zheng, J.; Chen, J.; Yan, Y. Correlating Hydrogen Oxidation and Evolution Activity on Platinum at Different $\mathrm{pH}$ with Measured Hydrogen Binding Energy. Nat. Commun. 2015, 6, 5848.

23. Zheng, J.; Sheng, W.; Zhuang, Z.; Xu, B.; Yan, Y. Universal Dependence of Hydrogen Oxidation and Evolution Reaction Activity of Platinum-Group Metals on $\mathrm{pH}$ and Hydrogen Binding Energy. Sci. Adv. 2016, 2, No. e1501602.

24. Cheng, T.; Wang, L.; Merinov, B.; Goddard, W. Explanation of Dramatic pH-Dependence of Hydrogen Binding on Noble Metal Electrode: Greatly Weakened Water Adsorption at High pH. J. Am. Chem. Soc. 2018, 140, 7787-7790. 
25. Ledezma-Yanez, I.; Wallace, W.; Sebastián Pascual, P.; Climent, V.; Feliu, J.; Koper, M. Interfacial Water Reorganization as a $\mathrm{pH}$ Dependent Descriptor of the Hydrogen Evolution Rate on Platinum Electrodes. Nature Energy 2017, 2, 1-7.

26. Liu, E.; Li, J.; Jiao, L.; Doan, H.; Liu, Z.; Zhao, Z.; Huang, Y.; Abraham, K.; Mukerjee, S.; Jia, Q. Unifying the Hydrogen Evolution and Oxidation Reactions Kinetics in Base by Identifying the Catalytic Roles of HydroxylWater-Cation Adducts. J. Am. Chem. Soc. 2019, 141, 3232-3239.

27. Skúlason, E.; Tripkovic, V.; Bjo“rketun, M.; Gudmundsdottir, S.; Karlberg, G.; Rossmeisl, J.; Bligaard, T.; Jónsson, H.; Nørskov, J. Modeling the Electrochemical Hydrogen Oxidation and Evolution Reactions on the Basis of Density Functional Theory Calculations. J. Phys. Chem. C 2010, 114, 1818218197.

28. Tan, C.; Cao, X.; Wu, X. -J.; He, Q.; Yang, J.; Zhang, X.; Chen, J.; Zhao, W.; Han, S.; Nam, G. -H.; Sindoro, M.; Zhang, H. Recent Advances in Ultrathin Two-Dimensional Nanomaterials. Chem. Rev. 2o17, 117, 6225-6331.

29. Guo, Y.; Xu, K.; Wu, C.; Zhao, J.; Xie, Y. Surface Chemical Modification for Engineering the Intrinsic Physical Properties of Inorganic Two-Dimensional Nanomaterials. Chem. Soc. Rev. 2015, 44, 637-646.

30. Fu, Q.; Han, J.; Wang, X.; Xu, P.; Yao, T.; Zhong, J.; Zhong, W.; Liu, S.; Gao, T.; Zhang, Z.;Xu, L.; Song, B. 2D Transition Metal Dichalcogenides: Design, Modulation, Challenges in Electrocatalysis. Adv. Mater. 2021, 33, 1907818.

31. Liu, Y.; Wu, J.; Hackenberg, K.; Zhang, J.; Wang, Y.; Yang, Y.; Keyshar, K.; Gu, J.; Ogitsu, T.; Vajtai, R. Self-Optimizing, Highly SurfaceActive Layered Metal Dichalcogenide Catalysts for Hydrogen Evolution. Nat. Energy 2017, 2, 17127.

32. Xi, Z. Underlying Structure-Activity Correlations of 2D Layered Transition Metal Dichalcogenides-Based Electrocatalysts for Boosted Hydrogen Generation. arXiv preprint 2021, arXiv: 2103. 02441.
33. Zhu, S.; Qin, X.; Yao, Y.; Shao, M. pH-Dependent Hydrogen and Water Binding Energies on Platinum Surfaces as Directly Probed Through Surface-Enhanced Infrared Absorption Spectroscopy. J. Am. Chem. Soc. 2020, 142, 8748-8754.

34. Jiao, Y.; Zheng, Y.; Jaroniec, M.; Qiao, S. Design of Electrocatalysts for Oxygen- and Hydrogen-Involving Energy Conversion Reactions. Chem. Soc. Rev. 2015, 44, 2060-2086.

35. Eng, A. Y. S.; Ambrosi, A.; Sofer, Z.; Simek, P.; Pumera, M. Electrochemistry of Transition Metal Dichalcogenides: Strong Dependence on the Metal-To-Chalcogen Composition and Exfoliation Method. ACS Nano 2014, 8, 12185 -12198.

36. Zhuang, P.; Sun, Y.; Dong, P.; Smith, W.; Sun, Z.; Ge, Y.; Ye, M. Revisiting the Role of Active Sites for Hydrogen Evolution Reaction Through Precise Defect Adjusting. Adv. Funct. Mater. 2019, 29, 1901290.

37. Li, D.; Wang, C.; Strmcnik, D.; Tripkovic, D.; Sun, X.; Kang, Y.; Chi, M.; Snyder, J.; van der Vliet, D.; Tsai, Y.; Stamenkovic, V.; Sun, S.; Markovic, N. Functional Links Between Pt Single Crystal Morphology and Nanoparticles with Different Size and Shape: The Oxygen Reduction Reaction Case. Energy Environ. Sci. 2014, 7, 4061-4069.

38. Deng, J.; Li, H.; Xiao, J.; Tu, Y.; Deng, D.; Yang, H.; Bao, X. Triggering the Electrocatalytic Hydrogen Evolution Activity of the Inert Two-Dimensional $\mathrm{MoS}_{2}$ Surface Via Single-Atom Metal Doping. Energy Environ. Sci. 2015, 8, 1594-1601.

39. Subbaraman, R.; Tripkovic, D.; Chang, K.; Strmcnik, D.; Paulikas, A.; Hirunsit, P.; Chan, M.; Greeley, J.; Stamenkovic, V.; Markovic, N. Trends in Activity for the Water Electrolyser Reactions on 3d M(Ni,Co,Fe,Mn) Hydr(oxy) oxide Catalysts. Nat. Mater. 2012, 11, 550557.

40. Wang, H.; Ming, M.; Hu, M.; Xu, C.; Wang, Y.; Zhang, Y.; Gao, D.; Bi, J.; Fan, G.; Hu, J. Size and Electronic Modulation of Iridium Nanoparticles on Nitrogen-Functionalized Carbon Toward Advanced Electrocatalysts for Alkaline Water Splitting. ACS Appl. Mater. Interfaces 2018, 10, 22340-22347. 
41. Garlyyev, B.; Kratzl, K.; Rück, M.; Michalička, J.; Fichtner, J.; Macak, J.; Kratky, T.; Günther, S.; Cokoja, M.; Bandarenka, A.; Gagliardi, A.; Fischer, R. Optimizing the Size of Platinum Nanoparticles for Enhanced Mass Activity in the Electrochemical Oxygen Reduction Reaction. Angew. Chem. Int. Ed. 2019, 58, 9596-9600.

42. Garlyyev, B.; Fichtner, J.; Piqué, O.; Schneider, O.; Bandarenka, A.; Calle-Vallejo, F. Revealing the Nature of Active Sites in Electrocatalysis. Chem. Sci. 2019, 10, 8060-8075. 